\title{
The time has come for
}

\section{Codman introduces ACCU-FLO* Shunt Systems,}

the result of a searching examination of available products, the advice of leading neurosurgeons, and our 135 years of experience in meeting surgical needs. We feel we can now make a fresh contribution to the management of hydrocephalus by offering a line of products integrated by their reliability.

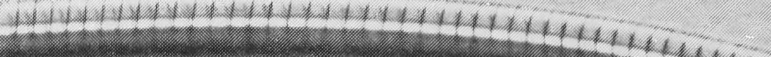

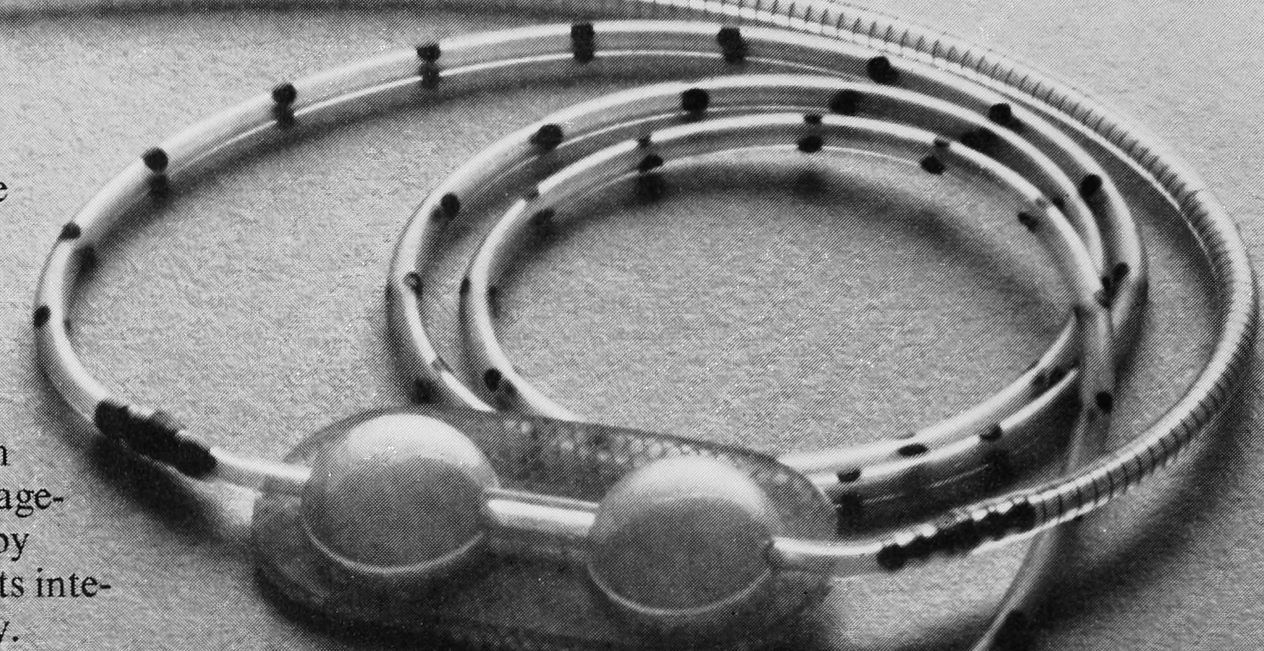

Cardiac and Peritoneal Catheters

The slits in our distal valve catheters are precisely cut by special equipment of our own design for more consistent opening and closing pressures. Available in low, medium, or high pressures, with radiopaque markers, barium impregnated, anti-kink imbedded spring, or open ended.

Reservoirs

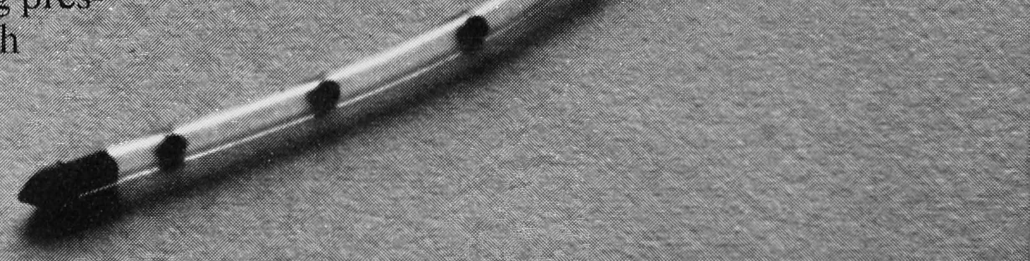

The bases of our reservoirs are designed to prevent a needle from passing through during medication. Available as double-dome flat bottom, single-dome flat bottom, or $14 \mathrm{~mm}$ burr hole.

\section{Ventricular Catheters}

Multiple small holes near the closed tips strain the C.S.F. to resist clogging. Available with radiopaque markers, barium impregnated, flanged barium impregnated, antikink imbedded spring, or right angle bend. 


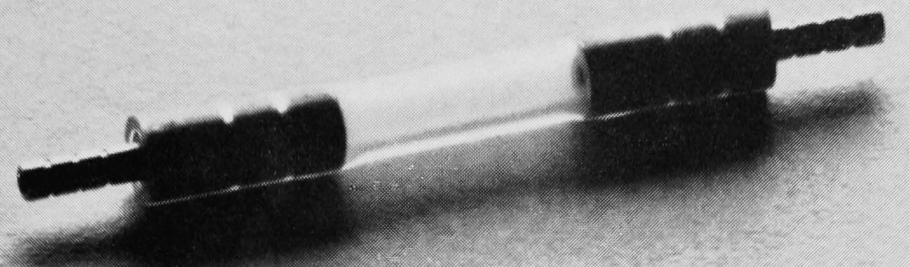

Our design incorporates two valves in one for increased reliability and double protection against refluxing. In addition, every ACCU-FLO Proximal Valve is delivered

with its own test report showing a strip-chart record of opening pressure, closing pressure, and flow rates. Available in low, medium, or high pressure.

"ACCU-FLO" means just what it says: Accurate flow. Since the treatment of hydrocephalus depends above all on the sure flow of C.S.F., we have made ACCU-FLO Shunt components as reliable as we know how. Ask us to send you our catalog of ACCU-FLO products and get a fresh look at dependability. Codman \& Shurtleff,

Division of Ethicon Sutures Ltd.,

Peterborough, Ontario K9J 7B9

\section{Codman*}




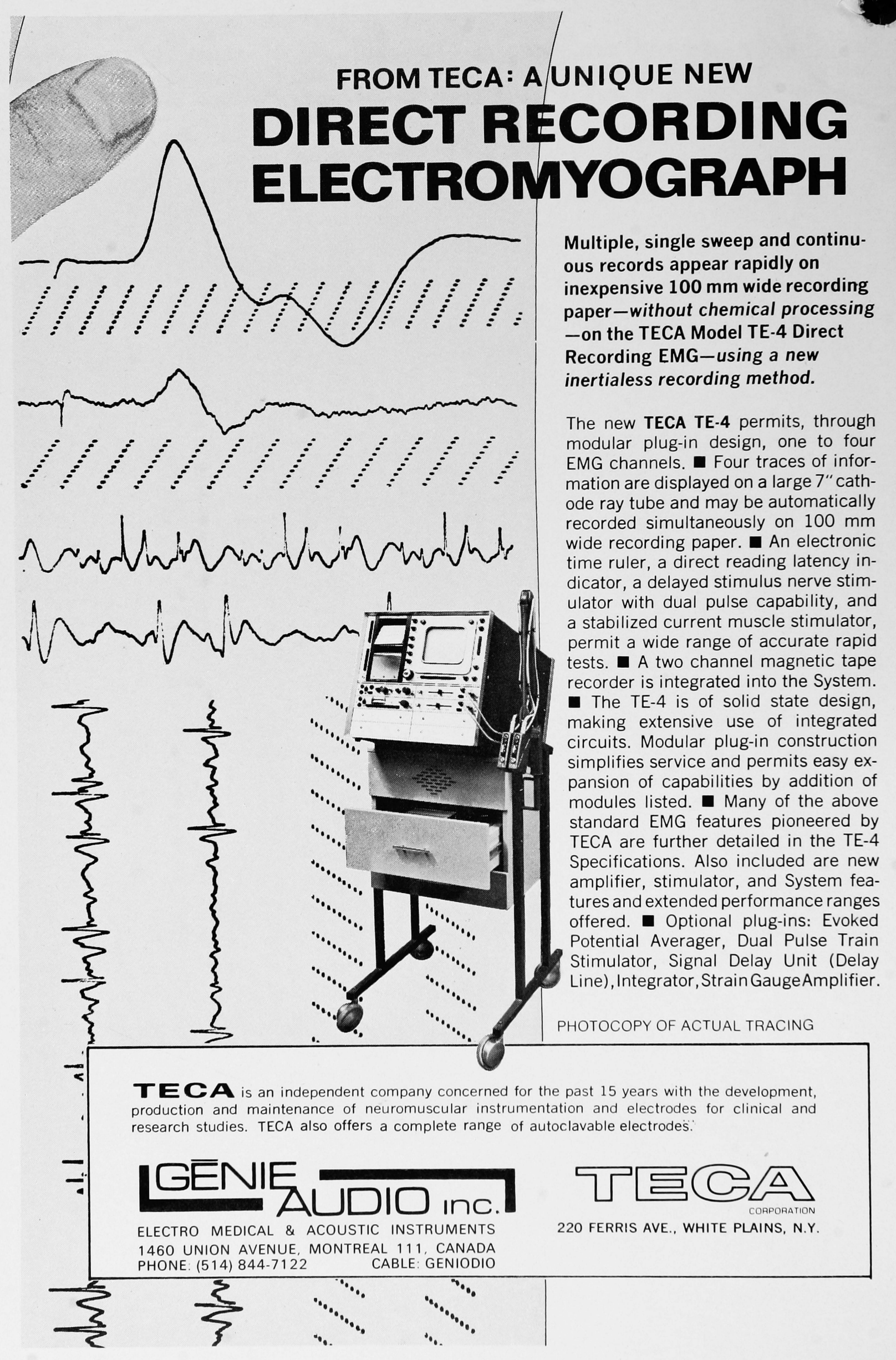




\section{New \\ 橉 Symmetre \\ for the management of Parkinson's syndrome \\ * Chemically distinct \\ (Not related to levodopa or anticholinergic \\ antiparkinson drugs \\ * Fast onset of action \\ (Usually effective within 1 week in contrast to \\ the slower response from levodopa)}

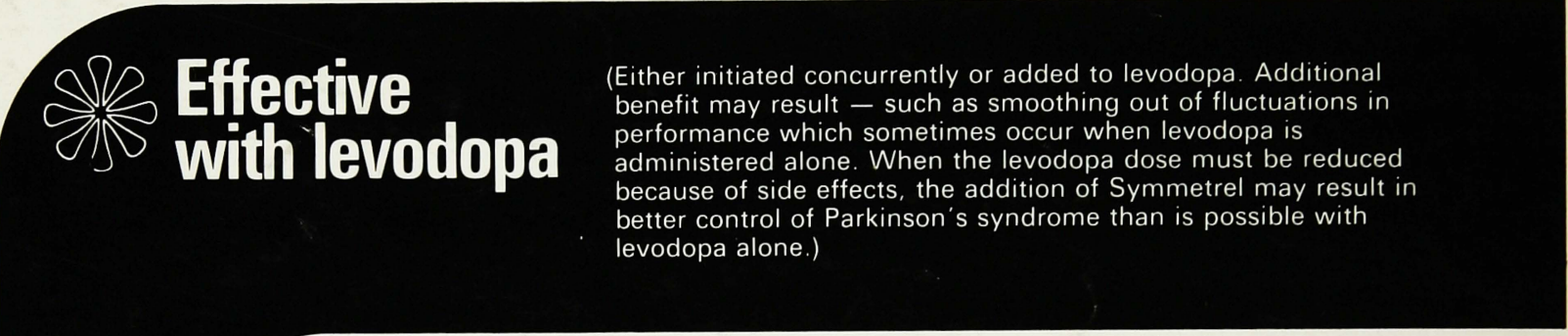 \\ Seffective with other anticholinergic \&s antiparkinson drugs}

When these drugs, e.g benztropine mesylate, provide only marginal benefits, Symmetrel used concomitantly may provide the same degree of control of Parkinson's syndrome, often with a lower dose of anticholinergic medication, and a possible reduction in anticholinergic side effects.) Effective alone

(Lessening of Parkinsonian symptomatology usually evident within one week in responsive patients.)

\section{CONTRAINDICATIONS}

WARNINGS Patients with a history of epilepsy or other "seizures" should be WARNINGS Por possible untoward central nervous system ellecis Patients with a history of congestive heart failure or peripheral edema should be receiving "Symmetrel" (amantadine HCI).
reveloped congesive heart latlure while Safety of use in pregnancy has not been established. Therefore "Symmetrel" should cian, the expected benefit to the patient outweighs the possible risks to the fetus Since the drug nursing mothers

PRECAUTIONS patients with renal impairment, congestive heart failure peripheral edema, or ortho
static hypotension. Since "Symmetrel" is not metabolized and is manly excreted in the urine, it may accumulate when renal function is inadequate. Care should be exercised when administering symmetrel to patients with iver or severe psychoneurosis not controlled by chemotherapeptic anents Casy abis or tion is required when "Symmetrel" is administered concurrently with central nervous

Patients with Parkinson's syndrome improving on "Symmetrel" should resume normal activities gradually and cautiously, consistent with other
tions. such as the presence of osteoporosis or phlebothrombosis Patients receiving "Symmetrel" (amantadine $\mathrm{HCl}$ ) who note central nervous system effects of blurring of vision should be cautioned against driving or working in situa Symmetrel (amantadine $\mathrm{HCl}$ ) should not be discontinued abruptly since a fow patients with Parkinson's syndrome experienced a Parkinsomian crisis, ie, sudden The dose of anticholnergic drugs or of "Symine 